\title{
New Ways towards a Solution of the False Alarm Problem
}

\author{
HANNES GUETTINGER and ANDREAS SCHEIDWEILER \\ Cerberus Ltd. \\ 8708 Maennedorf, Switzerland
}

ABSTRACT

The basic idea of the paper derives from the fact that the different activities of man is one of the most frequent sources of false alarms.

After the definition of the problem, today's counter-measures are described, i.e. the various attempts to improve the false alarm situation by developping proper standards and codes and designing better hard- and software.

The authors introduce an automatic fire detection system in which the fire sensitivity is controlled by so-called "presence detectors". This means in particular that depending on the presence or absence of man the fire detection sensitivity is automatically adapted.

First experimental results of this new concept are reported.

\section{INTRODUCTION}

There are no doubts that unwanted or false alarms have become a real nightmare for owners of automatic fire alarm systems and the fire brigade as well. As will be shown later, in most cases a human being was involved, i.e. directly or indirectly the fire originated from the activity of man.

However, man is not only a potential false alarm source, but also the best fire detector and fire fighter.

With our nose we are able to detect minute amounts of combustion products. Being a chemical sensor the capability of smelling lets us easily distinguish e.g. between smoke deriving from a burning steak or from an overloaded transformer. More than that it even detects the presence of certain gases. With our eyes we can see smoke and fire. We feel convected and radiated heat with our skin. Finally, we can also hear the noise created by the fire. Our brain combines all these inputs and enables us to make the distinction between friendly and dangerous or potentially dangerous fires. Being the result of so many criteria, the final decision is very reliable.

FIRE SAFETY SCIENCE-PROCEEDINGS OF THE SECOND INTERNATIONAL SYMPOSIUM, pD. 583-590 
Automatic fire detectors, on the other hand, usually still rely on the detection of a single fire criterion and a fixed alarm threshold. If this trigger level is exceeded by a deceptive phenomenon simulating a fire, the detector must respond with an alarm because there is no way it can distinguish between a harmless and a dangerous phenomenon. There is just not enough information available to make the right decision.

Therefore, attempts have been made to increase system intelligence by more sophisticated signal processing. This signal processing either is done in the detector or in the control panel.

Although progress has been reported, the problem of false alarm is still unsolved because the gap between the brain of a human being and that of a fire detection system remains too big.

\section{DEFINITION OF THE PROBLEM}

What are the main reasons for false alarms? An answer often heard is: "Too high sensitivity of smoke detectors".

Here, an important statement has to be made:

Smoke detectors, compared to heat detectors, really are intended for early warning. They are still the best and only early warning devices for the protection of life. It would mean to give up this most valuable advantage if we would solve the false alarm problem simply by reducing the sensitivity. Of course an unreasonably high sensitivity must be avoided. However, the price must not be a reduction of the protection value. Therefore, we have to increase the system intelligence.

A closer look to the different reasons for false alarms shows that they can be divided into four categories:

1. Alarms due to deceptive phenomena

2. Alarms due to unfavourable environmental conditions such as temperature, wind, electromagnetic interferences etc.

3. Alarms due to defective components

4. Alarms due to misoperation of the control panel (human error)

False alarms, traced back to category 2 and 3 are dealt with in the different standardization committees which try to develop new standards and codes.

Category 4 can be eliminated by a better design of the man-machineinterface of control panels.

There remains category 1 which is the subject of this paper. 
It is already quite a while that people in the industry realized, that false alarms become a problem if only a sufficient number of detectors is linked to a fire brigade. Countermeasures have been taken which have reduced the false alarm rate (ref. 1-3). These countermeasures helped for quite some time but in the future the requirements will be still higher.

Standards try to contribute to the reduction of false alarms by setting up requirements on quality and reliability in order to guarantee a stable response behaviour in the field. They also ask for a fire sensitivity which is a compromise between false alarm probability and early warning. Recently, the sensitivity requirements have been lowered in the US because of the bad false alarm situation.

Codes of practice giving clear guidelines concerning planning and installation of fire detection systems also help to avoid false alarms, in so far as general experience is concerned.

Furthermore, the manufacturers try to improve the situation with special instructions and concepts, custom tailored to their products. In particular, correct and regular maintenance in accordance with the manufacturers instructions could help to maintain a constant alarm threshold within a maintenance period.

In the last years, new systems have been introduced using the microprocessor technology available. This especially has improved the signal processing which goes so far that special algorithms (ref. 4,5) are applied in order to avoid false alarms.

In particular, the introduction of addressable systems made possible more economical solutions of already known concepts. The principle of crosszoning which certainly reduces false alarms is now possible without increased installation costs. Instead of combining zones, the concept of multi-detector dependence can be realized. Another proven technique is the so-called alarm verification which makes the alarm decision depending on how long the alarm threshold is surpassed. Here again, addressable systems have widened the application of this concept very much.

A step forward was furthermore the introduction of detectors which use two different sensons (criteria) connected in such a way that both of them must go into alarm (and-logic). Of course, such a logic combination reduces the fire sensitivity.

Last but not least, the detector design can help a lot to improve the situation. Appropriate entrance ports in order to minimize the influence of wind and dusting have created a relatively high resistance to false alarms due to these mentioned phenomena. Finally, the development of special labyrinths for optical smoke detectors have improved the susceptibility against false alarms caused by dust.

In this context the following method which has been applied successfully in many countries should be mentioned: 
Many years ago in Switzerland an alarm concept has been introduced which incorporates man into the alarm chain. The fact is taken into account that the intelligence of a human being could be a great help to answer the question whether there is a real fire or not. Instead of reducing the sensitivity the first alarm of an early warning detector is considered to be a warning as the name says. A well trained and instructed man is sent out to explore the situation. If he confirms that the warning was the early state of a real fire, he has the possibility to take appropriate countermeasures, i.e. to fight the fire or immediately call the fire brigade by pressing the alarm push button. Of course, the system allows the intervention of man only during a limited time. If the man does not react within that time the fire brigade is called automatically. During night time where the environment is much more "quiet" an alarm is immediately transmitted to the fire brigade (day/night setting of alarm system).

The philosophy behind that technique is the same that led to the proposed concept, $i$.e. alarms occuring during a time in which the fire detectors are subjected to deceptive phenomena produced by man should be evaluated before calling the fire brigade.

\section{NEW WAYS TOWARDS A SOLUTION}

Throughout the preceeding considerations we have seen that an effective reduction of the false alarm rate requires more information concerning deceptive phenomena. Although the described countermeasures have improved the situation, one must admit that, because of the increasing number of systems and the concentration of life and property in the big cities, the results are not satisfactory.

As already mentioned the proper choice of the alarm threshold is a very important decision during detector design. The following considerations shall show how the false alarm probability depends on the chosen sensitivity.

For the purpose of a simple model the stochastic response of a smoke sensor can be regarded as noise caused by many different sources of deceptive phenomena. Because there are many different sources, they can be considered statistically independent and the amplitude of the noise should show a normal distribution (ref. 6). In order to get an estimate of this distribution, averaging over a sufficient number of detectors and sufficient time is necessary. Our own measurements of the noise as well as measurements made by Siemens (ref. 6) justify this model (see fig. 1). The amplitude distribution can be written as

$$
p(A)=\frac{2}{\sqrt{2 \pi \sigma^{2}}} e^{-A^{2} / 2 \sigma^{2}}
$$

where $p(A)$ probability density

$$
\begin{aligned}
& \text { A amplitude of the sensor output } \\
& \sigma \text { variance of the amplitude distribution }
\end{aligned}
$$

The probability for false alarm $\mathrm{PFA}$ is then

$$
p_{F A}=p\left(A>A_{A l}\right)=\int_{A_{A l}}^{\infty} p(A) d A
$$




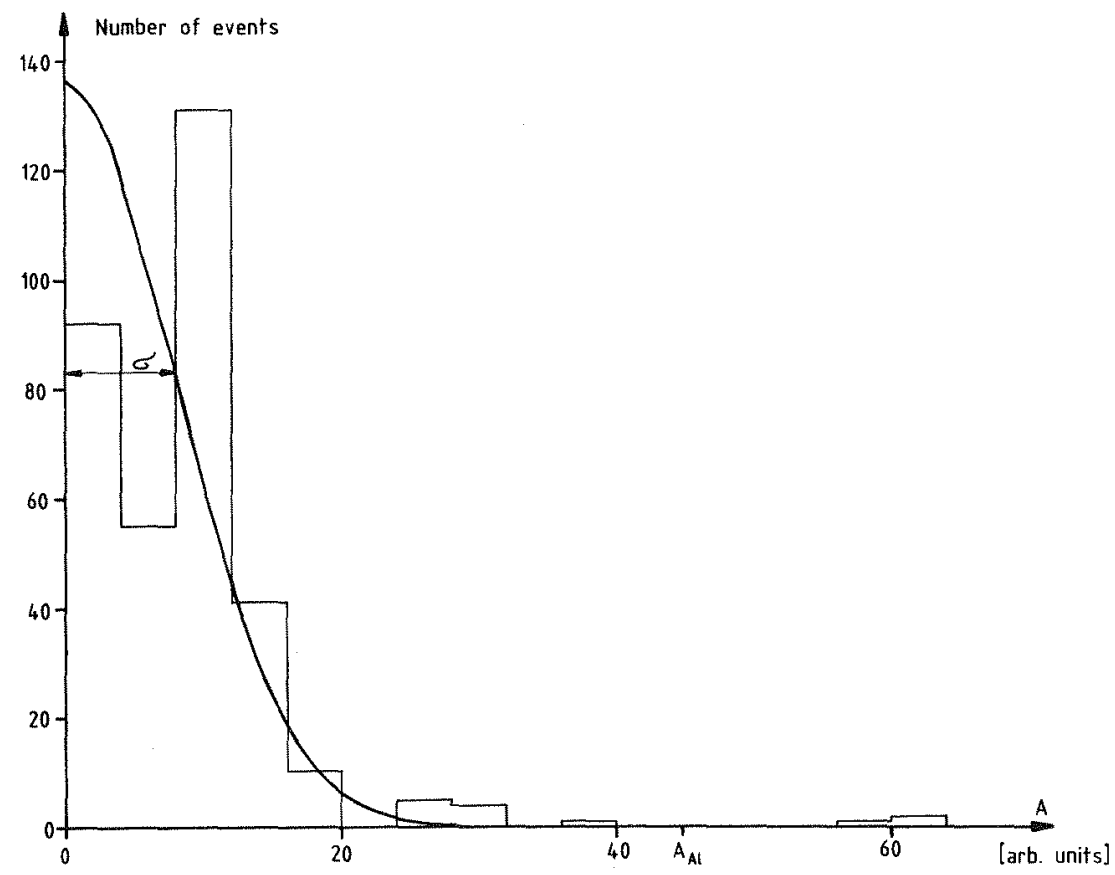

Fig. 1. Example of a measurement: Histogram of the signal magnitude of ionisation smoke detectors installed in an underground truck garage.

In the case of a system where the variance of the amplitude distribution stays below the alarm threshold $A_{A} I$ by a factor of three, $63 \%$ of all the events stay below $1 / 3 \mathrm{~A}_{\mathrm{Al}}$ and $93 \%$ below $\mathrm{A}_{\mathrm{Al}} / 2$. Lowering $\mathrm{A}_{\mathrm{Al}}$ by $10 \%$ raises the false alarm rate by a factor of 2.3 . From this example we see that we have a very powerful instrument in our hands if we allow changes in $\mathrm{AAI}$. The faise alarm rate can be held constant by adaption of $A_{A 1}$. In the field of radar-detection such adaptive threshold systems are known as CFAR (constant false alarm receiver). For a fixed threshold detector we see that setting the sensitivity as low as allowed to reliably detect all the dangerous fires will minimize the faise alarm rate. Intuition and experience already tell us this but the calculation makes clear how strongly the false alarm rate depends on the threshold level.

From this follows that it would be quite possible to adjust the detector sensitivity properly if the amplitude distribution of the noise signal would be known.

The proposed concept is based upon the assumption that in many cases a human being was the reason for false alarms. 


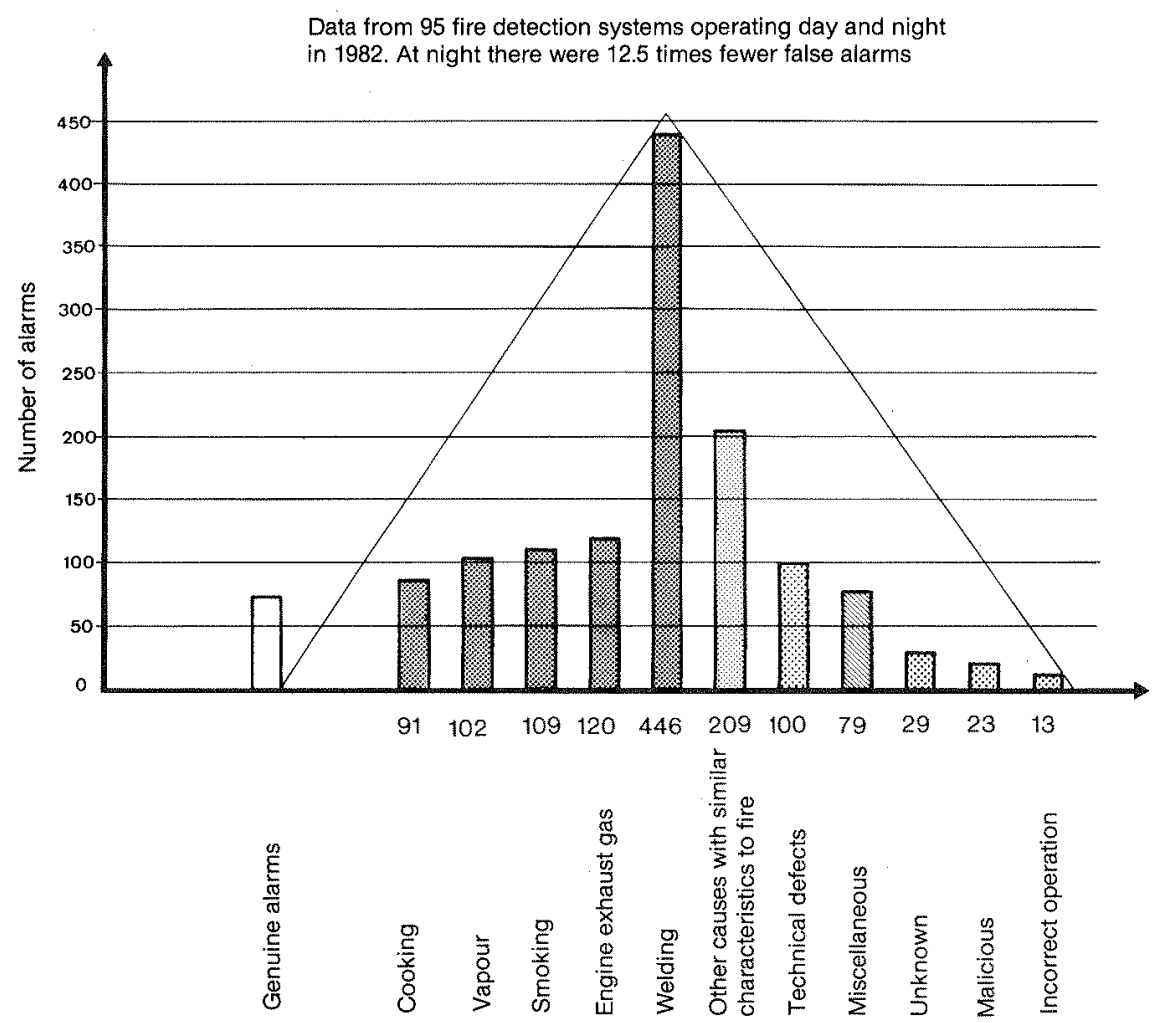

Fig. 2 Distribution of main problems in 95 systems - the "problem pyramid"

As can be seen from fig. 2 this hypothesis is justified because most of the deceptive phenomena such as cooking, smoking, welding and exhaust fumes from combustion engines really are caused by the activity of man (ref. 2). Thus, the presence of man and the occurrence of false alarms must be correlated. It is obvious that a reduction of the fire detector's sensitivity during the presence of man would reduce the false alarm rate because man as the primary source of false alarms is not active. Of counse, the reduction must stay within acceptable limits so that the detectors still give a minimum protection.

As an example, the following situation where the proposed concept could be applied successfully is considered:

In a conference room which unoccupied is a clean and quiet environment a smoke detector is installed. This detector supervises the empty room with a relatively high sensitivity (demanded by standards). If there is any smoke produced an immediate alarm is justified because most probably it is a fire.

On the contrary, if the room is filled with people who partly smoke, the sensitivity is too high. The result is a false alarm caused by smoking. 
With a smoke detector having a "people sensor" built-in or attached the problem could be solved to a large extent by applying the following alarm organisation:

In the empty state the "presence sensor", e.g. a passive infrared detector, would tell the smoke detector that the room is empty and the sensitivity is switched to "high" and an alarm is immediately transmitted to the fire brigade.

If the room is filled with people who are detected by the "presence sensor" the sensitivity is switched to "low", i.e. the alarm threshold is raised to a value which most probably is not reached by the respective deceptive phenomenon. This operation is justified because, in case of a real fire, there are enough living fire detectors available that can alarm. In particular the decision to call the fire brigade is left to man.

After the conference, i.e. after the "presence detector" has confirmed the "empty state" the sensitivity is switched back to "high", of course with a certain delay. If e.g. somebody has forgotten to extinguish his cigarette and a smoldering fire starts, the smoke detector will detect this danger with high sensitivity.

This example shows how, by automatically controlled sensitivity, false alarms can be avoided and the sensitivity is adjusted according to the noise level.

Other favorable applications for the proposed concept are:

- theaters

- working hal Is

- kitchens

- hotel jobbys

- office rooms

- etc.

Generally one can say that the concept is applicable in all cases of room protection with changing occupancy. Important is, however, that the people who are causing the sensitivity reduction are at least able to al arm, i.e. in hospitals or old people's homes the application is limited.

Concerning the choice of the "presence sensor" the passive infrared sensor of course is only one possibility. Another promising possibility is the use of a microphone which is able to detect the noise produced by man. A microphone has been installed at a truck delivery ramp where the trucks caused frequent false alarms of the optical smoke detectors. There was a high degree of correlation between "alarms" from the microphone and false alams of the optical smoke detectors.

Present work at CERBERUS concentrates on practical experience. Finally, it becomes a question of economics. In particular the question is investigated whether the "presence detector" is an integral part of the smoke detector or whether the "people detection" could be a separate system. In this context the combination between fire detection and intrusion systems will play an important role. 
Finally, the introduction of such a concept demands a reconsideration of the philosophy of standards and codes. With the present practice of the approval authorities which simply ask for the compliance with standards the permissible range in sensitivity is by far too small so that the expected success could be blocked. However, we are convinced that, if the results clearly show a breakthrough concerning the reduction of false alarms, the codes and regulations will have to be adapted.

\section{REFERENCES}

1. Watanabe, A., Sasaki, H. and Unoki, J., "Overview on Fire Detection in Japan", in Fire Safety Science edited by C.E. Grant and P.J. Pagni, Springer, 1985

2. Daetwyler, F., "False Alarm Reduction Programme FAREP", internal Cerberus publication, 1985

3. Bamert, E. and Neuenschwander, H., "Brandmeldeanlagen der neuen Generation: Technik, Ziele, Möglichkeiten", Brandverhutung 3/1987 p.6

4. Scheidweiler, A., "The distribution of intelligence in future fire detection systems", Fire Safety Journal, 6,209 (1983)

5. von Tomkewitsch, R., "Fire detection systems with distributed intelligence, the pulse polling system", Fire Safety Journal, 6, 225 (1983)

6. Thilo, P., Siemens Munich, private communication 